LAWRENCE LIVERMORE NAT IO N A L LABORATORY

\section{ELM-Induced Plasma Transport in the DIII-D SOL}

J.A. Boedo, D.L. Rudakov, E.M. Hollmann, R.A. Moyer, G.R. McKee, K. Burrell, T.E. Evans, A.W. Leonard, W.P. West, M.E. Fenstermacher, M. Groth, S.L. Allen, L. Zeng

January 11, 2005

16th International Conference on Plasma Surface Interactions Portland, ME, United States May 24, 2004 through May 28, 2004 
This document was prepared as an account of work sponsored by an agency of the United States Government. Neither the United States Government nor the University of California nor any of their employees, makes any warranty, express or implied, or assumes any legal liability or responsibility for the accuracy, completeness, or usefulness of any information, apparatus, product, or process disclosed, or represents that its use would not infringe privately owned rights. Reference herein to any specific commercial product, process, or service by trade name, trademark, manufacturer, or otherwise, does not necessarily constitute or imply its endorsement, recommendation, or favoring by the United States Government or the University of California. The views and opinions of authors expressed herein do not necessarily state or reflect those of the United States Government or the University of California, and shall not be used for advertising or product endorsement purposes. 


\title{
ELM-induced plasma transport in the DIII-D SOL
}

J.A. Boedo, ${ }^{a}$ D.L. Rudakov, ${ }^{a}$ E.M. Hollmann, ${ }^{a}$ R. A. Moyer, ${ }^{a}$ G.R. McKee, ${ }^{b}$ K. Burrell ${ }^{c}$, T. E. Evans, ${ }^{c}$ A.W. Leonard, ${ }^{c}$ W.P. West, ${ }^{c}$ M.E. Fenstermacher, ${ }^{d}$ M. Groth ${ }^{d}$ S.L. Allen, ${ }^{d}$ L. Zeng, ${ }^{e}$

G. Wang, ${ }^{e}$ J.G. Watkins, f and the DIII-D Team ${ }^{\mathrm{a}}$

${ }^{a}$ University of California, San Diego, La Jolla, California, USA

${ }^{b}$ University of Wisconsin, Madison, Wisconsin, USA

${ }^{c}$ General Atomics, P.O. Box, San Diego, California 92186-5608, USA

${ }^{d}$ Lawrence Livermore National Laboratory, Livermore, California, USA

${ }^{e}$ University of California Los Angeles, Los Angeles, California, USA

fSandia National Laboratories, Albuquerque, New Mexico, USA

\begin{abstract}
High temporal and spatial resolution measurements in the boundary of the DIII-D tokamak show that edge localized modes (ELMs) are composed of fast bursts of hot, dense plasma that travel radially starting at the separatrix at $\sim 450 \mathrm{~m} / \mathrm{s}$ and rotate in the scrape off layer (SOL), convecting particles and energy to the SOL and walls. The temperature and density in the ELM plasma initially correspond to those at the top of the density pedestal but decay with radius in the SOL. The temperature decay length $(\sim 1.2-1.5 \mathrm{~cm})$ is much shorter than the density decay length $(\sim 3-8 \mathrm{~cm})$, which in turn decreases with increasing pedestal density. The local particle and energy flux at the wall during the bursts are $10-50 \%$ $\left(\sim 1-2 ! \square ! 10^{21} ! \mathrm{m}^{-2} \mathrm{~s}^{-1}\right)$ and $1-2 \%\left(\sim 20-30 \mathrm{~kW} / \mathrm{m}^{2}\right)$ respectively of the LCFS average fluxes, indicating that particles are transported radially much more efficiently than heat.
\end{abstract}

JNM keywords: PO500, PO600

PSI-16 keywords: DIII-D, Edge Plasma, Cross-field transport, Intermittent transport

PACS: 52.40.Hf, 52.35.Ra, 52.55.FA, 52.70.Ds 
Corresponding author address: J.A. Boedo, UCSD, 9500 Gilman Dr., La Jolla, CA 920930417

Corresponding author e-mail: jboedo@ucsd.edu

Presenting author address: same as above

Presenting author e-mail: same as above

\section{Introduction}

High performance tokamak discharges operate in ELMing H-mode to combine highenergy confinement with adequate particle exhaust. The edge localized mode (ELM) instability also carries a considerable amount of particles and heat from the pedestal region into the scrape-off layer (SOL) towards the divertor region and other plasma facing components (PFCs), possibly limiting their lifetime and causing the release of impurities into the plasma. According to accepted scalings, Type I ELMs are expected to exceed the ITER PFC damage threshold (40-50 $\left.\mathrm{MJ} \mathrm{m}^{-2} \mathrm{t}^{-1}\right)$ by factors of 5 or more, ${ }^{1}$ resulting in a divertor lifetime of $<1$ full discharge! It is important to invest a significant effort to study ELMs the ELM dynamics in the SOL in order to envision ways of controlling their interaction with the PFCs.

A number of theoretical ELM studies ${ }^{2}$ have emphasized the linear regime of dominant modes $^{3}$ (coupled "peeling-ballooning" modes) are driven by parallel current $\left(J_{\text {ped }}\right)$ and the pressure gradient $(p$ ped $)$. These intermediate- $n$ peeling-ballooning modes, whose linear phase can be calculated using the ELITE code, impose limits on the pedestal height, which are functions of the pedestal width, plasma shape, collisionality, safety factor and other equilibrium details. Preliminary nonlinear studies ${ }^{4}$ suggest that during the ELM, filaments will grow in the pedestal region and travel across the separatrix into the SOL, carrying particles and heat with them. This paper provides information on ELM structure and propagation that can be used to improve the models.

\section{Experimental setup}

Experiments to characterize ELMs on the DIII-D tokamak ${ }^{5}$ were carried out in H-mode discharges featuring Type I ELMs with plasma current $I_{p} !=! 1.4$ !MA, toroidal field of 
$B_{T} !=! 1.7 ! \mathrm{T}$ at the axis, $R !=! 1.7 ! \mathrm{m}$ and neutral beam heating power of up to $4.5 \mathrm{MW}$. Lower single-null divertor geometry with ion Grad-B drift toward the lower divertor was used. The density was increased in a sequence of discharges, from $\hbar_{\mathrm{e}}\left\lceil\mathrm{n}_{\mathrm{G}}=0.40\right.$ to 0.8 . $\left(\mathrm{n}_{\mathrm{G}}\right.$ is the Greenwald limit) The principal measurements were made by the fast radiometer array 6 (DISRAD2), a fast scanning probe, ${ }^{7}$ Beam Emission Spectroscopy ${ }^{8}$ (BES), reflectometry 9 and $\mathrm{CER}^{10}$. The fast scanning probe array features five tips that sense current $I$, saturation current, $I_{s a t}$, and floating potential, $\square_{f}$. A $\approx ! 250 \mathrm{kHz}$ bandwidth $T_{e}$ measurement ${ }^{11}$ is used to resolve the temperature inside the ELMs. The BES system, configured as a $5 \square 6$ fiber array, was located at the edge of the plasma in the midplane to provide fast (1 $\square \mathrm{s}$ ) 2-D imaging of the density. The DISRAD2 measures absolutely calibrated photon intensity along 30 poloidally separated view chords at a rate of $100 ! \mathrm{kHz}$ and a photon energy response of 1 !eV to $5 ! \mathrm{keV}$.

\section{A. General ELM characterization}

Inversion of the DISRAD2 data (Fig. 1) shows the integrated ELM radiation for various plasma regions as a function of time measured from the beginning of an arbitrary ELM, or $t_{0}$. The radiation rises first on the outer SOL indicating low field side ELM formation, it then rises in the inner SOL and thereafter in the divertor. Two frames from BES taken at an arbitrary time near an ELM onset, $t_{0}^{\mathrm{BES}}$ and $16 \square \mathrm{s}$ later $\left(t_{0}^{\mathrm{BES}}+16 \square \mathrm{s}\right)$ and shown in Fig. !2, feature an ELM as a positive density feature appearing at the bottom of the frame and moving upward to the center. The data shown is the deviation from the average value and in the color scheme, white represents average (or background) density and red and blue represent positive and negative fluctuations, black indicates saturation. The LCFS is indicated by a solid vertical line. The ELM includes plasma ejections produced near the separatrix that then move radially at nearly $7 \mathrm{~km} / \mathrm{s}$ (the BES measurements were made in a different set of discharges that those otherwise discussed in this paper). Radial propagation into the SOL, seen by reflectometry ${ }^{12}$, shows ELMs with a velocity that peaks at $\sim 500 ! \mathrm{m} / \mathrm{s}$ and decays to $\sim 120 \mathrm{~m} / \mathrm{s}$ in the SOL. Probe measurements of $V_{r}=E_{\square} B / B^{2}$ agree with reflectometry 12 .

The ELMs have a complex spatio-temporal time structure, consistent with BES data, that is observed as multiple bursts in the temperature and density data taken by probes, shown in 
Fig.!3. The high time resolution data, displayed in a $12 ! \mathrm{ms}$ window that arbitrarily spans the ELM duration. The ELM plasma features peak $n_{e}$ values corresponding to those at the top of the density pedestal $\left(\sim 3 \square 10^{19} \mathrm{~cm}^{-3}\right)$ if the spatial decay is accounted for. The ELM peels off from the top of the density pedestal. The radial decay of the ELM peak density and temperature with distance from the LCFS varies with density shown in Fig.!4. At high $n_{e}$ $\left(n_{e} / n_{G}=0.85\right)$ the density decay length, $L_{N} ! \sim 3.8 \mathrm{lcm}$ while the temperature decay length, $L_{T}$ is $\sim 1.2 \mathrm{lcm}$. At $n_{e} / n_{G}=0.45, L_{N}$ and $L_{T}$ are $13 ! \mathrm{cm}$ and $1.3 ! \mathrm{cm}$, respectively. The particles in the ELM travel unhindered towards the wall at low densities while at high density, the particles are quickly dissipated. The heat in the ELM seems to be dissipated rapidly with radius, irrespective of the pedestal density, and consistent with IR camera results.

Probe and reflectometry data at the midplane indicate plasma convected at velocities of $\sim 450 \mathrm{!m} / \mathrm{s}$ that slow to $\sim 120 \mathrm{~lm} / \mathrm{s}$ into the SOL within $0.20 ! \mathrm{ms}$. At the highest speed, the ELM would strike the wall (6 to $7 ! \mathrm{cm}$ from the LCFS) in $\sim 0.15 ! \mathrm{ms}$, but due to the deceleration, the total radial transit time is closer to 0.3 !ms. The local peak heat and particle radial flux convected by the ELM towards the wall are $\square_{r}=n V_{r}$ and $Q_{r}=2 \square 3 / 2 n T V_{r}$ respectively. Due to bursts, the ELM-induced local convected radial heat flux at the LCFS is $\sim 80 \%$ of the average [calculated as $\left(\mathrm{P}_{\mathrm{in}}-\mathrm{P}_{\mathrm{rad}}\right) / \square_{\mathrm{E}} \mathrm{xArea}$ ] at $\square n_{e} \square n_{G W}=0.8$ and $\sim 60 \%$ at $\square n_{e} \square n_{G W}=0.45$. The heat flux reaching the wall (Table I), is only a $\sim 2 \%$ fraction of the LCFS heat flux, consistently with the short $(\sim 1.5 ! \mathrm{cm}) T_{e}$ radial decay, most of the heat flux is transported along the magnetic field and strikes the divertor floor. The ELM local convected radial particle flux due to the pulses at the wall is $10-50 \%$ of the LCFS local average radial flux, or $\sim 1.6 \square 10^{21} \mathrm{~m}^{\square 2} \mathrm{~s}^{\square 1}$, consistent with the long density decay length $(\sim 3-8 ! \mathrm{cm})$. Table!I indicates that the ELM local peak particle flux at the wall is quite similar for all densities due to the fact that at higher pedestal densities the ELM plasma density increases but the decay length becomes shorter.

\section{Interpretations of the ELM dynamics data}

Assuming mainly radial motion of the ELMs and using the measured radial ELM velocity, $V_{r}$, at the LCFS $(\sim 450 ! \mathrm{m} / \mathrm{s})$, the radial extent of each front is estimated as $\square r=V_{r} \square \square t$, where $\square t$ is the duration of the pulse. Radial extents of 2 to $5 ! \mathrm{cm}$ per pulse are obtained from the 
pulse duration ( $\sim 20$ to 40 ! $/ \mathrm{s}$ long). Gaps lasting $\sim 130$ to $140 \square \mathrm{s}$ are seen between pulses, sometimes followed by a longer gap of $\sim 800 \square \mathrm{s}$ between trains of pulses. The ELM comes from the LCFS in well-defined bursts that substantially fill the SOL (6 to 7 !cm gap) with dense plasma. This paradigm is hard to reconcile with the fact that many diagnostics in all toroidal and poloidal locations see the ELM promptly within a small time delay of $\sim 100 \square \mathrm{s}$.

Another possibility is that the ELM plasma is also rotating toroidally. The CER ${ }^{31}$ toroidal speeds during an ELM at radii straddling the LCFS are shown, together with a rotation profile [Fig. 5(b)] before $\left(t_{0}-1 \mathrm{~ms}\right)$ and during $\left(t_{0}\right)$ an ELM, where $t_{0}$ is determined by the rise of the fast $D_{\square}$ signal in the divertor. The onset of an ELM is concomitant with a transient increase of toroidal velocity and ion temperature (all from carbon) further out and into the SOL [Fig. 5(b,c)], which lasts «1 !ms, consistent with the radial ELM plasma travel time $(\sim 0.3 ! \mathrm{ms})$. The peak toroidal velocity and ion temperature of the radial transient corresponds to that of the innermost chord pre-ELM velocity, supporting that ELMs originate at the pedestal top. The CER-measured toroidal velocity is $\sim 30 ! \mathrm{km} / \mathrm{s}$ in the SOL, corresponds to $\sim 650 \square \mathrm{s}$ period, longer than the expected ELM plasma radial travel time of 200 to $300 \square \mathrm{s}$, so the ELM plasma does not have time to fully rotate before hitting the wall. Once the rotation assumption is considered, Fig. 3 can be re-examined. The initial density bursts are separated by $\sim 135 \square \mathrm{s}$, followed by a $\sim 450 \square \mathrm{s}$ gap before the bursts start again. This could be interpreted as three filaments rotating by the probe and returning with a period $T=800 \square \mathrm{s}$ while decaying by parallel transport.

\section{Acknowledgment}

This research was supported by the U.S. Department of Energy under DE-FC0204ER54698, *W-7405-ENG-48, DE-AC05-76OR00033, DE-AC04-94AL85000, DE-FG0204ER54758, DE-FG03-96ER54373, DE-FG03-01ER54615 and DE-AC04-94AL8500. The technical support of L. Chousal and R. Hernandez are acknowledged.

*This work was performed under the auspices of the U.S. Department of Energy by the University of California, Lawrence Livermore National Laboratory under Contract No. W-7405-Eng-48. 


\section{References}

[1] H.D. Pacher, et al., ITER Design Description Document, G 17 DDD 1 96-08-21 W2.1 (1996).

[2] J.W. Connor, et al., Phys. Plasmas 5 (1998) 2687.

[3] P.B. Snyder, et al., Phys. Plasmas 9 (2002) 2037.

[4] P.B. Snyder, X. Xu, private communication (2003).

[5] J.L. Luxon, Nucl. Fusion 42 (2002) 614.

[6] D.S. Gray, et al., "Time resolved radiated power during tokamak disruptions and spectral averaging of AXUV photodiode response in DIII-D," to be published in Rev. Sci. Instrum. (2003).

[7] J. Watkins, et al., Rev. Sci. Instrum. 63 (1992) 4728.

[8] R.J. Fonck, et al., Phys. Rev. Lett. 70 (1993) 3736.

[9] T.L. Rhodes, et al., Rev. Sci. Instrum. 63 (1992) 4599.

[10] K.H. Burrell, Rev. Sci. Instrum. 72 (2001) 906.

[11] D.L. Rudakov, et al., Rev. Sci. Instrum. 72 (2001) 453.

[12] L. Zeng, et al., Rev. Sci. Instrum. 74 (2003) 1530. 
Table 1. Radial particle $\left(T_{r}^{E L M}\right)$ and heat $\left(Q_{r}^{E L M}\right)$ fluxes due to an ELM at the LCFS and the wall for low density $\square n_{e} \square n_{G W}=0.45$ high density $\left\lceil n_{e} \square / n_{G W}=0.8\right.$ discharges

\begin{tabular}{|c|c|c|}
\hline $\begin{array}{c}\left\langle n_{\mathbb{T}}\right) m_{G W}=0.8 \\
\text { LCFS }\end{array}$ & $\begin{array}{c}\Gamma_{r}^{E L A}\left(\mathrm{~m}^{-2} \mathrm{~s}^{-1}\right) \\
1.0 \times 10^{22}\end{array}$ & $\begin{array}{c}\left.Q_{r}^{E L G} G \mathrm{~m}^{2} \mathrm{~s}^{-1}\right) \\
1_{0}, 80,000\end{array}$ \\
\hline Wall & $1.5 \times 10^{21}$ & 21,600 \\
\hline$\left\langle n_{2}\right)_{1} n_{C x}=0.45$ & $\Gamma_{g}^{E L A S}\left(\sin ^{-2} s^{-1}\right)$ & $Q_{\mathrm{g}}^{\text {ELs }}\left(\mathrm{U}_{\mathrm{m}} \mathrm{s}^{-2} \mathrm{~s}^{-1}\right.$ \\
\hline LCFS & $5.6 \times 10^{21}$ & $1,323,000$ \\
\hline Wall & $1.8 \times 10^{21}$ & 27,000 \\
\hline
\end{tabular}




\section{Figure captions}

Fig. 1 Inversion of DISRAD2 data from a single ELM (Type I) showing radiation peaking in the outer SOL, in the inner SOL, later at the inner divertor, and at the outer divertor, demonstrating ELM propagation over the top of the plasma into the inner divertor leg.

Fig. 2. Frames from BES showing 2-D density plots and taken every $1 \square \mathrm{s}$. The Type I ELM starts at $t_{0}$. A second frame is taken $16 \square \mathrm{s}$ thereafter to illustrate ELM characteristics. The ELM shows a poloidal structure that becomes quite complex. Radial and poloidal motion of ejecta is seen.

Fig. 3. High time resolution probe density data for a low discharge density $\left(n / n_{g}=0.4\right)$ showing the spatio-temporal complexity of ELMs. The successive bursts of high density lasts $\sim 30 \mathrm{Ls}$ and appear at fairly regular intervals $(\sim 140\llcorner\mathrm{~s})$. The intervals can show significant gaps of $\sim 300-400 ~ L s$ between groups of bursts.

Fig. 4. Radial variation of the ELM burst density and temperature values obtained from probes for (a) high density and (b) low density discharges. The temperature decays quickly with radius in both cases, but the density decay length is longer at low density.

Fig. 5. (a) CER data showing the toroidal rotation velocity of carbon ions for 7 radial positions inside the LCFS and in the SOL. A floor $D_{\square}$ signal [(a) top] is used as a fiducial. Profiles of rotation velocity (b) and ion temperature (c) taken during the ELM ( $\mathrm{t}=0$ ) and before the ELM ( $\mathrm{t}=-1)$ support the idea that the plasma at the top of the density pedestal is expelled into the SOL and rotates toroidally in the near SOL before being damped in the far SOL. 